\title{
Vantagens e limitações do uso do acesso intraósseo nas urgências e emergências: revisão integrativa
}

Advantages and limitations of the use of intra-bone access in emergencies and emergencies: integrative review Ventajas y limitaciones del uso del acceso intra-óseo en emergencias y emergencias: revisión integrativa

\begin{abstract}
RESUMO
Objetivo: Descrever as principais vantagens e limitações do uso do acesso intraósseo nas urgências e emergências. Métodos: Trata-se de uma revisão integrativa, que utilizou artigos disponibilizados na Biblioteca Virtual em Saúde, especificamente nas bases de dados da Literatura Latino-Americana e do Caribe em Ciências da Saúde e MEDLINE, e na Scientific Electronic Library Online, no período de agosto de 2016 a outubro de 2016. Resultados: Foram encontrados 292 artigos, e feita à seleção de 31 deles inicialmente. Após os critérios de exclusão, obteve-se como amostra final 09 artigos, agrupados de acordo com o autor, ano de publicação, título, objetivo, tipo de estudo e resultados. Conclusão: Ficou claro de que se trata de uma técnica de simples aprendizado e suas complicações são insignificantes. Demonstrou ser usado com segurança em diferentes locais de punção, tanto em adultos quanto crianças. Sendo assim, espera-se que este estudo possa suscitar novas pesquisas sobre a temática.

DESCRITORES: Emergências; Enfermagem em emergência; Infusões intraósseas; Métodos.
\end{abstract}

\section{ABSTRACT}

Objective: To describe the main advantages and limitations of using intraosseous access in urgencies and emergencies. Methods: This is an integrative review, which used articles available in the Virtual Health Library, specifically in the databases of Latin American and Caribbean Literature in Health Sciences and MEDLINE, and in the Scientific Electronic Library Online, in the period of august 2016 to october 2016. Results: 292 articles were found, and 31 of them were initially selected. After the exclusion criteria, 9 articles were obtained as a final sample, grouped according to the author, year of publication, title, objective, type of study and results. Conclusion: It was clear that this is a simple learning technique and its complications are insignificant. It has been shown to be used safely in different puncture sites, both in adults and children. Therefore, it is hoped that this study may prompt further research on the subject.

DESCRIPTORS: Emergencies; Emergency nursing; Intraosseous infusions; Methods.

\section{RESUMEN}

Objetivo: Describir las principales ventajas y limitaciones de utilizar el acceso intraóseo en urgencias y emergencias. Métodos: Es una revisión integradora, que utilizó artículos disponibles en la Biblioteca Virtual en Salud, específicamente en las bases de datos de Literatura Latinoamericana y Caribeña en Ciencias de la Salud y MEDLINE, y en la Biblioteca Científica Electrónica en Línea, en el período de agosto de 2016 a octubre de 2016. Resultados: Se encontraron 292 artículos, de los cuales 31 fueron seleccionados inicialmente. Tras los criterios de exclusión, se obtuvieron 9 artículos como muestra final, agrupados según autor, año de publicación, título, objetivo, tipo de estudio y resultados. Conclusión: quedó claro que esta es una técnica de aprendizaje simple y sus complicaciones son insignificantes. Se ha demostrado que se utiliza de forma segura en diferentes sitios de punción, tanto en adultos como en niños. Por lo tanto, se espera que este estudio impulse más investigaciones sobre el tema. DESCRIPTORES: Emergencias; Enfermería de emergencia; Infusiones intraóseas; Métodos.

RECEBIDO EM: 29/08/2020 APROVADO EM: 23/10/2020

\section{Kamilla Carla Ferreira}

Enfermeira. Graduada em Enfermagem pela Universidade de Itaúna (UIT), Minas Gerais.

ORCID: 0000-0002-5840-1435 


\section{Thayane Vieira Carvalho}

Enfermeira. Pós graduada em Oncologia Multiprofissional pelo Instituto Israelita de Ensino e Pesquisa Albert Einstein, São Paulo.

ORCID: 0000-0001-8644-8954

\section{Marla Ariana Silva}

Graduanda em Enfermagem pela Universidade do Estado de Minas Gerais (UEMG), Unidade Divinópolis. ORCID: 0000-0003-0136-7122

\section{Allan de Morais Bessa}

Graduando em Enfermagem pela Universidade do Estado de Minas Gerais (UEMG), Unidade Divinópolis. ORCID: 0000-0001-6969-7537

\section{Vinícius Silva Belo}

Biólogo. Mestre em Epidemiologia em Saúde Pública pela Fundação Oswaldo Cruz (FIOCRUZ). Doutor em Epidemiologia em Saúde Pública pela Escola Nacional de Saúde Pública (ENSP). Docente da Universidade Federal de São João Del Rei (UFSJ), campus Centro-Oeste. Docente e coordenador do programa de Pós-Graduação em Ciências da Saúde. Orientador de mestrado e de doutorado.

ORCID: 0000-0003-0183-1175

\section{Silmara Nunes Andrade}

Enfermeira. Doutora em Ciências da Saúde pela Universidade Federal de São João Del Rei (UFSJ), Campus Centro Oeste Dona Lindu (CCO). Docente do Curso de Graduação em Enfermagem da Universidade do Estado de Minas Gerais (UEMG), Unidade Divinópolis.

ORCID: 0000-0002-1975-0827

\section{Regina Consolação dos Santos}

Enfermeira. Mestre em Ciência pela Universidade Federal de São João Del Rei (UFSJ), Campus Centro Oeste Dona Lindu (CCO). Docente do Curso de Graduação em Enfermagem da Universidade do Estado de Minas Gerais (UEMG), Unidade Divinópolis.

ORCID: 0000-0002-7393-3210

\section{Heber Paulino Pena}

Enfermeiro. Mestre em Enfermagem. Docente da Faculdade de Enfermagem da Universidade de Itaúna - Minas Gerais. ORCID: 0000-0002-9122-6827

\section{INTRODUÇÃO}

V ivemos em constante evolução, usando a tecnologia a favor da melhoria das condições de saúde da população. Os atendimentos de urgência e emergência têm se destacado pelo grande número de atendimentos dos mais variados tipos. Aponta-se que, nas regiões metropolitanas, as principais causas de morte, na faixa etária entre 15 e 49 anos, são traumas por acidentes, casos de envenenamento e violências ${ }^{(1-2)}$. Atualmente, são gastos mais de seis bilhões de reais em serviços hospitalares e ambulatoriais, e mais de um bilhão no suporte profilático e terapêutico, no $\mathrm{Brasil}^{(3)}$. $\mathrm{O}$ estado de Minas Gerais apresenta um gasto de cerca de 354 milhões de reais executados anualmente em urgência e emergência, destacando as regiões do Sul de Minas, que gasta mais de 79 milhões, e a região Central, com gasto de mais de 75 milhões de reais, segundo pesquisas realizadas em $2015^{(4)}$.

A obtenção de uma via para administração de fluídos e medicamentos é indispensável nos atendimentos de urgências e emergências, e acessos como a via intravenosa periférica podem não ser possíveis de serem obtidos dependendo de diversos fatores que envolvem o paciente. Outra opção também seria o acesso por via central, porém não é recomendado devido à demora de se estabelecê-lo ${ }^{(5-7)}$.

Devido à facilidade e ao risco mínimo de complicações, a via intravenosa periférica continua sendo a primeira opção em casos de urgência, e após não estabelecida com três tentativas em curto prazo segundo as últimas Diretrizes da American
Heart Association (AHA) sobre Ressuscitação Cardiopulmonar (RCP) e Atendimento Cardiovascular de Emergência (ACE), a via intraóssea é a segunda mais eficiente opção, por ser um procedimento rápido que dura em média 10 à 20 segundos e suas contra indicações serem apenas a presença de fratura ou outros traumas na inserção local, articulação prostética, tentativas de punções anteriores, osteoporose ou outras anormalidades, como por exemplo, infecção no local ${ }^{(8-9)}$.

Descrito inicialmente em 1922 por Drinker $^{(10)}$, foi substituído por Josefson ${ }^{(11)}$ em 1934, nas emergências pediátricas. $\mathrm{Na}$ década de 1940, passou a ser usada com mais frequência devido a Segunda Guerra Mundial, caindo, logo após, em desuso, com o avanço tecnológico e o surgimento de novos cateteres ${ }^{(12-13)}$. Em 1980 voltou a 
ganhar destaque, sendo usados com mais eventualidade em crianças e adultos, tendo em vista que o acesso intraósseo fornece uma via rígida, não colapsável na cavidade medular dos ossos longos, para infusão de fluídos e medicamentos ${ }^{(14)}$.

Há diferentes dispositivos para a realização do acesso intraósseo disponíveis comercialmente de fácil uso. Os mais usados são o Driver (EZ-IO), o BIG e o Fast (dispositivo de impacto) que funciona como uma arma de injeção. $\mathrm{O}$ EZ-IO, aprovado pela Food and Drug Administration em 2004(15), tem sido utilizado recentemente em ambiente pré-hospitalar ${ }^{(16)}$, podendo ser usado com diferentes comprimentos de agulhas e medidores para a colocação em crianças e adultos $^{(17)}$. Apesar da facilidade do acesso e de possuir o material necessário, os estudos sobre a via intraóssea destaca, principalmente, a preocupação das inserções pré-hospitalares e a falta de habilidades necessárias para realizar tal procedimento ${ }^{(18)}$.

Os conselhos responsáveis pela fiscalização das atividades profissionais do enfermeiro considerem que o mesmo possui competência legal para realizar procedimento de punção intraóssea, porém, considera que a realização do procedimento de punção intraóssea é considerada um procedimento complexo relacionado ao contexto de atendimento em situações de urgência e emergência, reconhecendo ser imprescindível a sua capacitação técnica. Assim, considera-se lícito que em situações de urgência e emergência e visando a preservação da vida, o profissional enfermeiro realize o procedimento de punção intraóssea ${ }^{(14)}$.

Nesta perspectiva, o presente estudo acarretará importantes contribuições a esta categoria profissional, refletindo na execução com maior segurança do procedimento de punção intraóssea e aumentando consequentemente, a qualidade e a eficácia do cuidado adulto e pediátrico. Tendo em vista a relevância do assunto abordado, o objetivo deste estudo foi descrever as principais vantagens e limitações do uso do acesso intraósseo nas urgências e emergências.

\section{MÉTODOS}

Refere-se a um estudo de revisão integrativa, realizada a partir de levantamentos bibliográficos em bases de dados acadêmicos, que busca fornecer uma análise efetiva de resultados a fim de contribuir para o tema abordado e para a prática. É caracterizado por cinco etapas, sendo: a identificação da questão da pesquisa; estabelecimento de critérios de inclusão e exclusão; análise dos dados; avaliação dos estudos e apresentação dos resultados ${ }^{(19)}$.

No geral, para a construção da revisão integrativa é preciso percorrer seis etapas distintas, similares aos estágios de desenvolvimento de pesquisa convencional ${ }^{(19)}$. Visto isso, foram utilizadas as seguintes etapas para análise e seleção dos artigos: formulação da pergunta norteadora: (Quais vantagens e limitações do uso do acesso intraósseo na urgência e emergência?); critérios para inclusão e exclusão de estudos; definição das informações a serem extraídas dos estudos selecionados; avaliação dos estudos incluídos na revisão integrativa; interpretação dos resultados; apresentação da revisão.

A busca na base de dados foi realizada no período de agosto de 2016 a outubro de 2016. O estudo foi composto por artigos da internet e foram selecionados artigos da literatura internacional, publicados em português, inglês ou espanhol, por meio dos resumos disponibilizados na Biblioteca Virtual em Saúde (BVS), especificamente nas bases de dados da Literatura Latino-Americana e do Caribe em Ciências da Saúde (LILACS) e MEDLINE, e na Scientific Electronic Library Online (SciELO) segundo o protocolo Preferred Reporting Itens for Systematic Reviews and Meta-Analyses (PRISMA) ${ }^{(19)}$.

A seleção dos estudos foi realizada por dois pesquisadores, em buscas independentes. Em caso de desacordo, um terceiro autor foi consultado e a decisão final foi tomada por consenso. Com o propósito de garantir rigor metodológico, consultaram-se os DeCS (Descritores em Ciências e Saúde) para a seleção dos termos, sendo definida como equação de busca a seguinte combinação: Pregnancy (Unplanned OR Unwanted) AND Breastfeeding.

Os descritores utilizados foram ("Infusions, Intraosseous/adverse effects" [Mesh] OR "Infusions, Intraosseous/contraindications" [Mesh] OR "Infusions, Intraosseous/history" [Mesh] OR "Infusions, Intraosseous/instrumentation" [Mesh] OR "Infusions, Intraosseous/ methods" [Mesh] OR "Infusions, Intraosseous/nursing" [Mesh] OR "Infusions, Intraosseous/organization and administration" [Mesh] OR "Infusions, Intraosseous/pharmacology” [Mesh] OR "Infusions, Intraosseous/standards" [Mesh] OR "Infusions, Intraosseous/ statistics and numerical data" [Mesh] OR "Infusions, Intraosseous/therapeutic use" [Mesh] OR "Infusions, Intraosseous/therapy" [Mesh] OR "Infusions, Intraosseous/trends" [Mesh] OR "Infusions, Intraosseous/veterinary” [Mesh]) AND ("Infusions, Intraosseous/adverse effects" [Majr:noexp] OR "Infusions, Intraosseous/contraindications" $\quad[\mathrm{Ma}$ jr:noexp] OR "Infusions, Intraosseous/ history" [Majr:noexp] OR "Infusions, Intraosseous/instrumentation” [Majr:noexp] OR "Infusions, Intraosseous/ methods" [Majr:noexp] OR "Infusions, Intraosseous/nursing” [Majr:noexp] OR "Infusions, Intraosseous/standards" [Majr:noexp] OR "Infusions, Intraosseous/ statistics and numerical data" [Majr:noexp] OR "Infusions, Intraosseous/trends" [Majr:noexp] OR "Infusions, Intraosseous/veterinary" [Majr:noexp]).

Foram utilizados os seguintes critérios de inclusão: artigos em português, espanhol ou inglês; artigos originais, disponíveis e gratuitos, que continham um ou mais descritores, publicação o período de 2006 a 2016 e que se enquadravam ao tema abordado. Foram excluídos os artigos repetidos, que foram publicados antes do ano 2006, que não se enquadravam ao tema escolhido.

Como estratégia de busca dos artigos foi utilizada a ferramenta de PICO, que é um instrumento que baseia em evidências científicas. O método PICO pode ser utilizado para construir temas de pesquisa de 


\section{artigo}

Ferreira C.F.; Carvalho, R.V.; Ariana Silva, M.; Bessa, A.M.; Belo, V.S.; Andrade, S.N.; Santos, R.C.; Pena, H.P.; Vantagens e limitações do uso do acesso intraósseo nas urgências e emergências: revisão integrativa

\section{Quadro 1 - Descrição da estratégia Pico - Itaúna, Minas Gerais, 2016}

\begin{tabular}{|c|c|c|}
\hline Acrônimos & DESCRIÇÃo & ANÁLISE \\
\hline P & Paciente & $\begin{array}{c}\text { Pacientes vítimas de situações de urgências e } \\
\text { emergências }\end{array}$ \\
\hline I & Intervenção ou indicador & Acesso Intraósseo \\
\hline C & Comparação ou controle & Não se aplica \\
\hline O & Outcomes - desfecho & $\begin{array}{c}\text { Evidenciar as vantagens e limitações do uso do } \\
\text { acesso intraósseo nas urgências e emergências }\end{array}$ \\
\hline \multicolumn{2}{|l}{ Fonte: Rev. Latino-am Enfermagem, 2007ºl. } \\
\hline
\end{tabular}

\section{Quadro 2 - Critérios de Exclusão}

\begin{tabular}{|c|c|}
\hline CRITÉRIO DE EXCLUSÃO & QUANTIDADE DE ARTIGOS EXCLUÍDOS \\
\hline Artigos Repetidos & 04 \\
\hline Artigos Incompletos & 05 \\
\hline Artigos que não atenderam aos objetivos & $\begin{array}{c}\text { 08, sendo 03 destes sobre estudos em } \\
\text { animais. }\end{array}$ \\
\hline $\begin{array}{c}\text { Artigos que os dados já haviam sido analisa- } \\
\text { dos em outras referências }\end{array}$ & 05 \\
\hline Fonte: Autores, 2016. \\
\hline
\end{tabular}

naturezas diversas, originárias da clínica, do gerenciamento de recursos humanos e materiais, da procura de ferramentas para avaliação de sintomas, entre outras. A pergunta de pesquisa apropriada (bem construída) permite a definição adequada de que informações (evidências) são imprescindíveis para a resolução da questão clínica de pesquisa ${ }^{(20)}$. A síntese dos dados através da estratégia de PICO é demonstrada a seguir no Quadro 1 .

Por meio deste método de busca, foram encontrados 292 artigos, e feita à seleção de 31 deles inicialmente. Foram então realizados os critérios de exclusão, conforme descrito abaixo no Quadro 2.

\section{RESULTADOS}

Após a análise, obteve-se como amostra final 08 artigos, agrupados conforme mostra a seguir no Quadro 3.

\section{Quadro 3 - Referências incluídas na revisão integrativa}

\begin{tabular}{|c|c|c|c|c|}
\hline $\mathbf{N}^{\circ}$ & TÍTULO & $\begin{array}{l}\text { AUTOR/ANO DE } \\
\text { PUBLICAÇÃO }\end{array}$ & TIPO DE ESTUDO & RESULTADOS \\
\hline 1 & Intraosseous Access & $\begin{array}{c}\text { Hans Rosenberg BSc MD, } \\
\text { Warren James Cheung BHSc } \\
\text { MD / } 2013\end{array}$ & $\begin{array}{l}\text { Estudo observa- } \\
\text { cional }\end{array}$ & $\begin{array}{l}\text { Dois lactentes foram admitidos na } \\
\text { unidade neonatal com dificuldade } \\
\text { venosa, foram ressuscitados com êxito } \\
\text { de episódios de colíose usando o acesso } \\
\text { intraósseo. }\end{array}$ \\
\hline 2 & $\begin{array}{l}\text { Intraosseous Emergency Access } \\
\text { by Physicians Wearing Full } \\
\text { Protective Gear }\end{array}$ & $\begin{array}{c}\text { Ron Bem - Abraham, MD, } \\
\text { Ilan Gur, MD, Youri Vater, } \\
\text { MD, and A. Weinbroum, MD. } \\
\text { / } 2003\end{array}$ & $\begin{array}{c}\text { Estudo } \\
\text { Experimental }\end{array}$ & $\begin{array}{l}\text { A aplicação d BIG foi testada } 20 \text { vezes, } \\
\text { tendo sucesso em } 80 \% \text { das tentativas } \\
\text { realizadas de forma rápida e segura, } \\
\text { obtendo resultado eficaz. }\end{array}$ \\
\hline 3 & $\begin{array}{l}\text { Resuscitation in Massive } \\
\text { Obstretic Haemorrhage Using } \\
\text { Intraosseous Needle }\end{array}$ & $\begin{array}{l}\text { D. J. Chatterjee, B. Bukunda, } \\
\text { T. L. Samuels, L. Induruwage } \\
\text { and D. R. Uncles / } 2011\end{array}$ & Relato de Caso & $\begin{array}{l}\text { O sucesso do acesso neste caso } \\
\text { baseou-se em um elemento chave do } \\
\text { processo inicial de ressuscitação, que foi } \\
\text { a utilização da agulha intraóssea. }\end{array}$ \\
\hline 4 & $\begin{array}{l}\text { Recommendations for the use } \\
\text { of intraosseous vascular access } \\
\text { for emergent and noneemer- } \\
\text { gent situations in various health } \\
\text { care settings: a consensus pa- } \\
\text { pel. Crit Care Nurse. }\end{array}$ & $\begin{array}{l}\text { Phillips L, Brown L, Campbell } \\
\text { T, Miller J, Proehl J, Youn- } \\
\text { gberg B. / } 2010 .\end{array}$ & $\begin{array}{l}\text { Análise de Lite- } \\
\text { ratura }\end{array}$ & $\begin{array}{l}\text { A evolução tecnológica dos dispositi- } \\
\text { vos intraósseos torna o procedimento } \\
\text { relativamente fácil de se executar com } \\
\text { educação e formação adequadas. }\end{array}$ \\
\hline 5 & $\begin{array}{l}\text { Intraosseous Devices for Intra- } \\
\text { vascular Access in Adult Trauma } \\
\text { Patients }\end{array}$ & $\begin{array}{c}\text { Michael W. Day, RN, MSN, } \\
\text { CCRN / } 2011\end{array}$ & $\begin{array}{l}\text { Estudo Compa- } \\
\text { rativo }\end{array}$ & $\begin{array}{c}\text { A via intraóssea foi consistentemente } \\
\text { ser um meio rápido e confiável de ob- } \\
\text { tenção do acesso vascular, no sucesso } \\
\text { da ressuscitação. }\end{array}$ \\
\hline
\end{tabular}




\begin{tabular}{|c|c|c|c|c|}
\hline 6 & $\begin{array}{l}\text { An Observational, Prospective } \\
\text { Study to Determine the Ease of } \\
\text { Vascular Access in Adults Using } \\
\text { a Novel Intraosseous Access } \\
\text { Device }\end{array}$ & $\begin{array}{c}\text { Ong, Marcus EH, Adeline SY } \\
\text { Ngo, and Ramesh Wijaya / } \\
2009\end{array}$ & $\begin{array}{l}\text { Estudo Observa- } \\
\text { cional }\end{array}$ & $\begin{array}{l}\text { Os resultados dos dispositivos mesmos } \\
\text { com suas limitações vivenciadas foram } \\
\text { de sucesso, comprovando o tempo } \\
\text { médio de colocação do BIG em } 100 \% \text { e } \\
\text { El-Zo em } 87 \% \text {. }\end{array}$ \\
\hline 7 & $\begin{array}{c}\text { A theoretical alternative intra- } \\
\text { osseous infusion site in severely } \\
\text { hypovolemic children }\end{array}$ & $\begin{array}{l}\text { Mogale, Nkhensani, Alber- } \\
\text { t-Neels Van Schoor, and } \\
\text { Marius C. Bosman / } 2015\end{array}$ & $\begin{array}{l}\text { Estudo de caso } \\
\text { com exploração } \\
\text { anatômica em } \\
\text { cadáveres. }\end{array}$ & $\begin{array}{l}\text { Com base nos dados anatômicos cole- } \\
\text { tados, é provável que seja uma alterna- } \\
\text { tiva segura, quando a agulha é inserida } \\
\text { conforme o método discutido. }\end{array}$ \\
\hline 8 & $\begin{array}{l}\text { Complicación de la vía intraósea } \\
\text { en un neonato }\end{array}$ & Carreras González, E. / 2012 & Estudo de Caso & $\begin{array}{c}\text { A punção intraóssea é a via de escolha } \\
\text { mais segura e apropriada em relação } \\
\text { à ressuscitação infantil, quando se } \\
\text { desempenha um papel importante em } \\
\text { permanecer por um tempo mínimo } \\
\text { possível. }\end{array}$ \\
\hline
\end{tabular}

\section{DISCUSSÃO}

Os textos encontrados foram lidos, organizados e sintetizados em 05 categorias temáticas, a saber: Acesso Intraósseo Pediátrico; Acesso Intraósseo no Adulto, Locais e Tipos de Dispositivos; Competências do Médico e do Enfermeiro.

\section{Acesso intraósseo pediátrico}

Em 1922, Drinker já propunha conforme estudos, o uso do acesso intraósseo em recém-nascidos e crianças, devido situações de riscos e procedimentos apropriados sem complicações. A tecnologia e estudos atuais permitem que a infusão em via intraóssea seja considerada a forma mais segura e eficaz, quando comparado com falhas ou impossibilidades de acessos intravenosos, centrais e umbilical ${ }^{(21)}$. Percebe-se a dificuldade da infusão devido a não inserção do acesso periférico ${ }^{(22)}$, ou mesmo a resistência devido ao calibre de pequeno Lúmen para um fluxo rápido ${ }^{(23)}$.

Com isso descobriram que, com o acesso intraósseo, fluidos injetáveis entram com uma taxa muito mais satisfatória na circulação. O local de primeira escolha é o terço superior da tíbia ou o terço do fêmur, devido não haver músculos intervindo, o osso esterno não deve ser escolhido em crianças de 03 a 05 anos, por ter maior risco de atingir o coração ou outros órgãos ${ }^{(24)}$. Sendo essas estruturas ósseas firmes, a agulha não se desloca, mesmo com movimentos excessivos do paciente ou transporte pré-hospitalar, não havendo desconforto na infusão lenta, apenas se o fluído for numa pressão maior, onde pode haver um aumento da pressão intramedular ${ }^{(23-25)}$.

Portanto, sabemos da precisão de uma via mais segura e disponível de administração de fluidos em crianças e os ossos longos indicam uma via de sucesso, não afetando o crescimento dos mesmos e oferecendo facilidade, agilidade, segurança e poucas complicações que não são consideradas objeções para o não uso desse método ${ }^{(24)}$.

\section{Acesso intraósseo no adulto}

Assim como é realizado o procedimento pediátrico, a utilização da via intraóssea nos adultos foi utilizada amplamente durante a segunda guerra mundial em 1940, caindo em desuso até a década de 1980,quando passou a ser usada novamente com mais frequência ${ }^{(21)}$, sendo que esta via proporciona um efeito significativo que é determinante em casos de suporte de vida avançado ${ }^{(27-28)}$ onde a inserção do acesso é feita através de ossos longos (úmero, tíbia, fêmur, esterno),que tem uma cavidade com maior concentração de medula óssea e não é constrita ${ }^{(21,26-28)}$.

Com a análise dos estudos feitos pela AHA e da associação internacional de reanimação, o acesso Intraósseo é recomendado como uma intervenção padrão após as ten- tativas de métodos tradicionais de acessos intravenosos como os periféricos, jugulares e centrais, de difíceis ou impossíveis obtenções, sendo que são separadas de acordo com a prioridade do paciente ${ }^{(23,29)}$.

A inserção do acesso intraósseo é feita em um prazo menor que um minuto e pode ser administrado em um fluxo de aproximadamente $125 \mathrm{ml}$ por minuto de fluídos, e medicamentos reanimadores como atropina, epinefrina e adrenalina com a mesma dosagem de quando são feitos intravenosos, podem ser feitos também hemoderivados como plasmas, insulinas, sulfonamidas (antimicrobianos), penicilina (antibióticos), antitoxinas, e ainda análises de amostras sanguíneas ${ }^{(23,}$ 24). O tempo de uso do acesso é de 24 horas, o que é necessário para a reposição volêmica e estabilidade para punção de uma via intravenosa ${ }^{(23)}$.

Porém não se pode esquecer-se dos riscos e perturbações que são apresentadas no momento do procedimento, como dores, infecções ou sangramento, que não devem ser excluídos como queixas nos adultos, porém a eficácia deve ser considerada como um dos melhores métodos benéficos já estudados para o tratamento de doentes e para redução de risco iminentes ${ }^{(21,28)}$.

\section{Locais e tipos de dispositivos}

Foram aprovados três dispositivos intraósseos pelo Food And Drug Administration para o uso em pacientes com traumas 
onde o acesso intravenoso exige muito tempo para inserção ou é totalmente impossível devido ao estado hipovolêmico ${ }^{(23)}$.

O BIG (BIG injection gun) pode ser usado na tíbia e úmero proximal, seu manuseio é feito por uma pressão manual, dispositivo pequeno e leve, disponível em cores azul para adultos e vermelho para crianças, com profundidade de penetração recomendada de $2,5 \mathrm{~cm}$ para o adulto, para crianças de 0 a 3 anos de $0,5 \mathrm{~cm}$ à $0,7 \mathrm{~cm}$, pediátrico de 3 à 6 anos de $1,0 \mathrm{~cm}$ à $1,5 \mathrm{~cm}$, e o pediátrico de 6 à 12 anos de $1,5 \mathrm{~cm}$. Possui boa fixação, o que facilita no transporte de pacientes, fabricado em Israel pela WAISMED, chegou ao Brasil em 2007. Consiste em um equipamento com uma mola comprida que é disparada após retirar sua trava, possui ainda um regulador para o local de aplicação ou por faixa etária e é um dispositivo descartável ${ }^{(23,29)}$.

O EZ-IO pode ser usado na tíbia proximal, tíbia distal e úmero proximal, é um equipamento do tipo pistola (furadeira) funcionando por bateria com conjunto de três agulhas com tamanhos distintos e descartáveis. $\mathrm{O}$ dispositivo pode ser esterilizado após o uso, apresenta-se grande diferencial sobre a inserção onde há excesso de tecidos, com boa fixação. Fabricado nos Estados Unidos da América pela empresa VIDA CAREN ${ }^{(23,29)}$.

O FAST 1 é usado restritamente na região do osso esterno, local que pode exigir a utilização das duas mãos para exercer a pressão necessária. É ativado por uma aplicação manual, dispositivo leve e pequeno, fabricado no Canadá pela empresa PYNG MEDICAL. Possui um introdutor com agulhas diversas para aplicação ${ }^{(23,29)}$.

\section{Competências do médico e do en- fermeiro}

Após estudos dos artigos, foi analisado que os profissionais médicos e enfermeiros são habilitados a realizar a técnica da punção intraóssea, porem pouco usam devido ainda ao desconhecimento existente sobre o mesmo ${ }^{(28)}$.

Os médicos são mais propensos a realizar tal procedimento, pois possuem conhecimento criterioso de indicação e vantagens. Uma de suas intervenções é a mudança de comportamento e o reconhecimento do enfermeiro como um profissional habilitado na condução da técnica e das demais situações emergenciais. $\mathrm{O}$ enfermeiro, por sua vez, tem a capacitação necessária para realizar o atendimento ao paciente, usando, se for o caso, o acesso intraósseo como via escolhida na situação, onde contribui para prevenção de atrasos no atendimento ${ }^{(8,28)}$.

Segundo a Resolução do Conselho Regional de Enfermagem de São Paulo (Coren-SP) a Punção Intraóssea por Enfermeiros é competência do médico e do enfermeiro, que atuam em serviços de urgências e emergências, a execução deste procedimento, caso necessário, de forma segura e rápida, propiciando um ganho de tempo no atendimento e melhorias no prognóstico do paciente. O CTA 006.95 considera favorável a realização da técnica por enfermeiros em emergências pediátricas, e novos estudos científicos comprovaram a realização do mesmo também em adultos, permitindo assim ser realizado pelo enfermeiro, desde que este esteja devidamente treinado e que exerça seu papel com competência, honestidade, justiça e responsabilidade, assegurando sempre uma assistência de enfermagem de qualidade e sem danos ${ }^{(14)}$.

O profissional ainda deve garantir que a instituição em que atua, possua protocolos que assegurem todas as suas ações perante cada atendimento prestado, que ambos sejam seguidos de forma correta por toda a equipe e que todas as ações realizadas sejam registradas nos prontuários de forma clara e objetiva, conforme descrito em seu código de ética ${ }^{(8,28)}$.

Podemos concluir que o enfermeiro capacitado é habilitado a realizar a técnica do acesso intraósseo em qualquer situação emergenciais, desde que possua treinamento adequado, para assim garantir um melhor atendimento ao paciente.

\section{CONCLUSÃO}

O acesso intraósseo é utilizado para garantir de uma forma mais rápida via de administração eficaz de fluidos e medicamentos quando há algum obstáculo na obtenção do acesso venoso periférico. Essa técnica do acesso venoso intraósseo necessita ser difundida e mais utilizada, pois é um procedimento simples e de fácil realização. A intervenção rápida na dificuldade de acesso venoso em situações de emergências na maioria das vezes é um determinante para a manutenção da vida humana.

Sendo assim, espera-se que este estudo possa suscitar novas pesquisas sobre a temática, bem como demonstrar aos enfermeiros que atuam na Urgência e Emergência sobre seu importante papel no acesso venoso intraósseo e o prognóstico em uma situação real torna-se mais favorável.

\section{REFERÊNCIAS}

1. Brasil. Ministério da Saúde. Secretaria Executiva. Urgência e Emergência: Sistemas Estaduais de Referências Hospitalar para o Atendimento de Urgência e Emergência. - Brasília: Ministério da Saúde, 2001. 28p. Disponível em: https://bvsms.saude.gov. br/bvs/publicacoes/urgencia_emergencia.pdf. Acesso em: 10 jul. 2020.

2. Dejours C. Normalidade, trabalho e cidadania. Cadernos do CRP. $1991 ; 6(1): 13-7$.
3. Brasil. Ministério da Saúde. Portal da Saúde. Despesas com Saúde por Subfunção. Brasília, DF. 2016. Disponível em: http:// www.portaltransparencia.gov.br/funcoes/10-saude?ano=2020. Acesso em: 10 jul. 2020.

4. Minas Gerais. Plano Estadual de Saúde. 2016. Disponível em: https://www.conass.org.br/pdf/planos-estaduais-de-saude/ MG_Plano-Estadual-de-Sade-2016-2019-Final-aprovado.pdf. Acesso em: 20 jul. 2020. 


\section{REFERÊNCIAS}

5. Halm B, Yamamoto LG. Comparing ease of intraosseous needle placement: Jamshidi versus Cook. Am J Emerg Med 1998; 16:4201. Doi: https://doi.org/10.1016/S0735-6757(98)90146-2.

6. Jun H, Haruyama AZ, Chang KS, Yamamoto LG. Comparision of a new screw tipped intraosseous needle versus a standard bone marrow aspiration needle for infusion. Am J EmergMed 2000;18:135-9. Doi: https://doi.org/10.1016/S07356757(00)90003-2.

7. Calkins MD, Fitzgerald G, Bentley TB, Burris D. Intrassoues infusion devices: a comparison for potential use in special operations. J Trauma 2000; 48:106874. Doi: 10.1097 / 00005373200006000-00012.

8. Cheung WJ., Rosenberg H.; Vaillancourt C. Barriers and facilitators to intraosseous access in adult resuscitations when peripheral intravenous access is not achievable. Academic Emergency Medicine. 2014, 21.3: 250-256. Doi: https://doi.org/10.1111/acem.12329.

9. Selby IR., James MR. The Intraosseous route for induction of anaesthesia. Volume 48. 1993. Doi: https://doi. org/10.1111/j.1365-2044.1993.tb07480.x.

10. Drinker CK, Drinker KR, Lund CC. The circulation inthe mammalian bone marrow. Am J Physiol, 1922; 62:1-92. Doi: https:// doi.org/10.1152/ajplegacy.1922.62.1.1

11. Josefson A. A new method of treatment intraossal injections. Acta MedScad, 1934; 81-550-564. Doi: https://doi. org/10.1111/j.0954-6820.1934.tb19683.x.

12. Tocantins LM. Rapid absorption of substances injected in the bone marrow. The American Journal of Medicine, 1940;45:292296. Doi: https://doi.org/10.1016/0002-9343(51)90039-3.

13. Tocantins LM, O'Neill JF, Jones HW. Infusions of blood and other fluids via the bone marrow applications in pediatrics. JAMA, 1941;117:1229-1234. Doi: https://doi.org/10.1001 / jama.1941.02820410007002.

14. Conselho Regional de Enfermagem de São Paulo. Realização de punção intraóssea por enfermeiros. São Paulo. 2009. Disponível em: https://portal.coren-sp.gov.br/wp-content/uploads/2015/07/Parecer\%2001\%20-\%202009\%20atualizado_0. pdf. Acesso em: 20 jul. 2020.

15. Blumberg SM, Gorn M, Crain EF. Intraosseous infusion: a review of methods and novel devices. Pediatric Emergency Care. 2008; 24:50-9. Doi: https://doi.org/10.1097/pec.0b013e31815f727b.

16. Glaeser PW, Hellmich TR, Szewczuga D. Five-year experience in prehospital intraosseous infusions in children and adults. Ann Emerg Med. 1993;22:1119-24. Doi: https://doi.org/10.1016/ S0196-0644(05)80975-8.

17. Franscone RJ, Jensen J, Wewerka SS, Salzman JG. Use of the pediatric EZ-IO needle by emergency medical services providers. Pediatric Emergency Care. 2009;25:329-32. Doi: https://doi. org/10.1097 / PEC.0b013e3181a341fa.

18. Derinoz O, Keles A. Department of Pediatrics Emergency. Powered Intraosseous Device (EZ-IO) for Critically III Patients.
Pediatri Indiano. 2013; 50 (7): 689-91. Doi: 10.1007 / s13312013-0192-z.

19. Mendes KDS, Silveira RCCP, Galvão CM. Revisão Integrativa: método de pesquisa para a incorporação de evidências na saúde e na enfermagem. Texto Contexto Enferm, Florianópolis, 2008 Out-Dez; 17(4): 758-64. Doi: http://dx.doi.org/10.1590/S010407072008000400018.

20. Santos CMC, Pimenta CAM, Nobre MRC. A estratégia Pico para a construção da pequena pergunta de pesquisa e busca de evidências. Ver Latino-am Enfermagem 2007; 15(3). Doi: https:// doi.org/10.1590/S0104-11692007000300023.

21. Mogale N, Schoor ANV, Bosman MC. A theoretical alternative intraosseous infusion site in severely hypovolemic children. African journal of primary health care \& family medicine, 2015 . Vol 7, N 1: 1-5. Doi: https://doi.org/10.4102/phcfm.v7i1.835.

22. Chatterjee DJ, Bukunola B, Samuels TL, Induruwage L, Uncles DR. Resuscitation in massive obstetric haemorrhage using an intraosseous needle. Anaesthesia 66.4. 2011; 306-310. Doi: https://doi.org/10.1111/j.1365-2044.2011.06629.x.

23. Day MW. Intraosseous devices for intravascular access in adult trauma patients. Critical care nurse 31.2. 2011; 76-90. Doi: https://doi.org/10.4037/ccn2011615.

24. Ramet J, Slaats MALJ, Elsing CJ. Intraosseous Infusions in Infants and Neonates. Em: Bar-Shalom D., Rose K. (eds) Pediatric Formulations. AAPS Advances in the Pharmaceutical Sciences Series. Springer, New York. 2014. Doi: https://doi. org/10.1007/978-1-4899-8011-3_21.

25. Carreras-González E, Brió-Sanagustín S, Guimerá I, Crespo C. Complicación de la vía intraósea en un neonato. Med. Intensiva. 2012; 36(3):233-234. Doi: https://doi.org/10.1016/j.medin.2011.05.004.

26. Rosenberg $\mathrm{H}$, Cheung WJ. Intraosseous access. Canadian Medical Association Journal 185.5. 2013; E238-E238. Doi: https://doi.org/10.1503/cmaj.120971.

27. Ben Abraham R, Gur I, Vater $Y$, Weinbroum AA. Intraosseous emergency access by physicians wearing full protective gear. Academic emergency medicine 10.12. 2003; 1407-1410. Doi: https://doi.org/10.1111/ j.1553-2712.2003.tb00019.x.

28. Phillips L, Brow L, Campbell T, Miller J, Proehl J, Youngberg B. Recommendations for the use of intraosseous vascular access for emergent and nonemergent situations in various health care settings: a consensus paper. Critical care nurse 30.6. 2010: e1e7. Doi: https://doi.org/10.4037/ccn2010632.

29. Ong ME, Ngo AS, Wijaya R. An observational, prospective study to determine the ease of vascular access in adults using a novel intraosseous access device. Annals Academy of Medicine Singapore. 2009 Feb;38(2):121-4.

30. Tan BK, Chong S, Koh ZX. EZ-IO in the ED: na observational, prospective study comparing flow rates with proximal and distal tibia intraosseous acess in adults. Am J. Emerg Med 2012, jan 11. Doi: https://doi.org/10.1016 / j.ajem.2011.10.025. 